\title{
A STUDY OF CLOUD COMPUTING AND ITS ARCHITECTURE
}

Mustafa Muhasili

BCA student

Department of Computer Science

Kalinga University, Naya Raipur, Chattisgarh, India

\begin{abstract}
Cloud Computing, a rapidly developing information technology has aroused the concern of the whole world. Cloud computing is Internet-based computing, whereby shared resources, software and information are provided to computers and devices on demand. Cloud computing is the product of the fusion of traditional computing technology and network technology like grid computing, distributed computing, parallel computing and so on. It aims to construct a perfect system with powerful computing capability through a large number of relatively low-cost computing entity, and using the advanced business models like SaaS (Software As A Service), PaaS (Platform As A Service), IaaS (Infrastructure As A Service) to distribute the powerful computing capacity to end users.

Despite all the hype surrounding the cloud, enterprise customers are still reluctant to deploy their business in the cloud. Security is one of the major issues which reduces the growth of cloud computing and complications with data privacy and data protection continue to plague the market. Most of the security and privacy issues in cloud computing are caused by users lack of control over the physical infrastructure.

Many Cloud Service Providers (CSP) offer access to scalable, reliable computing resources following a pay-asyou-go model. Research into the security of the Cloud focuses mainly on protecting legitimate users of Cloud Services from attacks by external, malicious users. Little attention is being given to prohibit malicious users from using the Cloud to launch attacks, such as those currently done by botnets. These attacks include launching a DDoS attack, sending spam and perpetrating click fraud.
\end{abstract}

Keywords - Cloud computing, cloud storage, cloud service model, Type of cloud, cloud architecture

\section{INTRODUCTION}

\subsection{Cloud Computing}

The NIST (National Institute of Standards and Technology) definition of cloud computing states that:

\author{
Rahul Kumar Chawda \\ Assistant Professor \\ Department of Computer Science \\ Kalinga University, Naya Raipur, Chattisgarh, India
}

Cloud Computing is a model for enabling ubiquitious, convenient, on-demand network access to a shared pool of configurable computing resources (e.g., networks, servers, storage, applications, and services) that can be rapidly provisioned and released with minimal management effort or service provider interaction. This cloud model promotes availability and is composed of five essential characteristics, three service models and four deployment models.

\section{Essential Characteristics:}

- On-Demand Self-Service: A consumer can unilaterally provision computing capabilities, such as server time and network storage, as needed automatically without requiring human interaction with each service's provider.

- Broad Network Access : Capabilities are available over the networka and accessed through mechanisms that promote use by heterogenous thin or thick client platforms (e.g., mobile phones, laptops and PDAs).

- Resource Pooling : The provider's computing resources are pooled to serve multiple consumers using a multitenant model, with different physical and virtual resources dynamically assigned and reassigned according to consumer demand. There is a sense of location independence in that the customer generally has no control or knowledge over the exact location of the provided resources but may be able to specify location at a higher level of abstraction (e.g., country, state or datacenter). Examples of resources include storage, processing, memory, network bandwidth, and virtual machines.

- Rapid Elasticity: Capabilities can be rapidly and elastically provisioned, in some cases automatically, to quickly scale out, and rapidly released to quickly scale in. To the consumer, the capabilities available for provisioning often appear to be unlimited and can be purchased in any quantity at any time.

- Measured Service: Cloud Systems automatically control and optimize resource use by leveraging a metering capability at some level of abstraction appropriate to the type of service (e.g., storage, processing, bandwidth, and active user accounts). Resource usage can be monitored, 
controlled, and reported, providing transparency for both the provider and consumer utilized service.

\section{LITERATURE REVIEW}

Cloud Architecture, the systems architecture of the software systems involved in the delivery of cloud computing, typically involves multiple cloud components communicating with each other over application programming interfaces, usually web services.

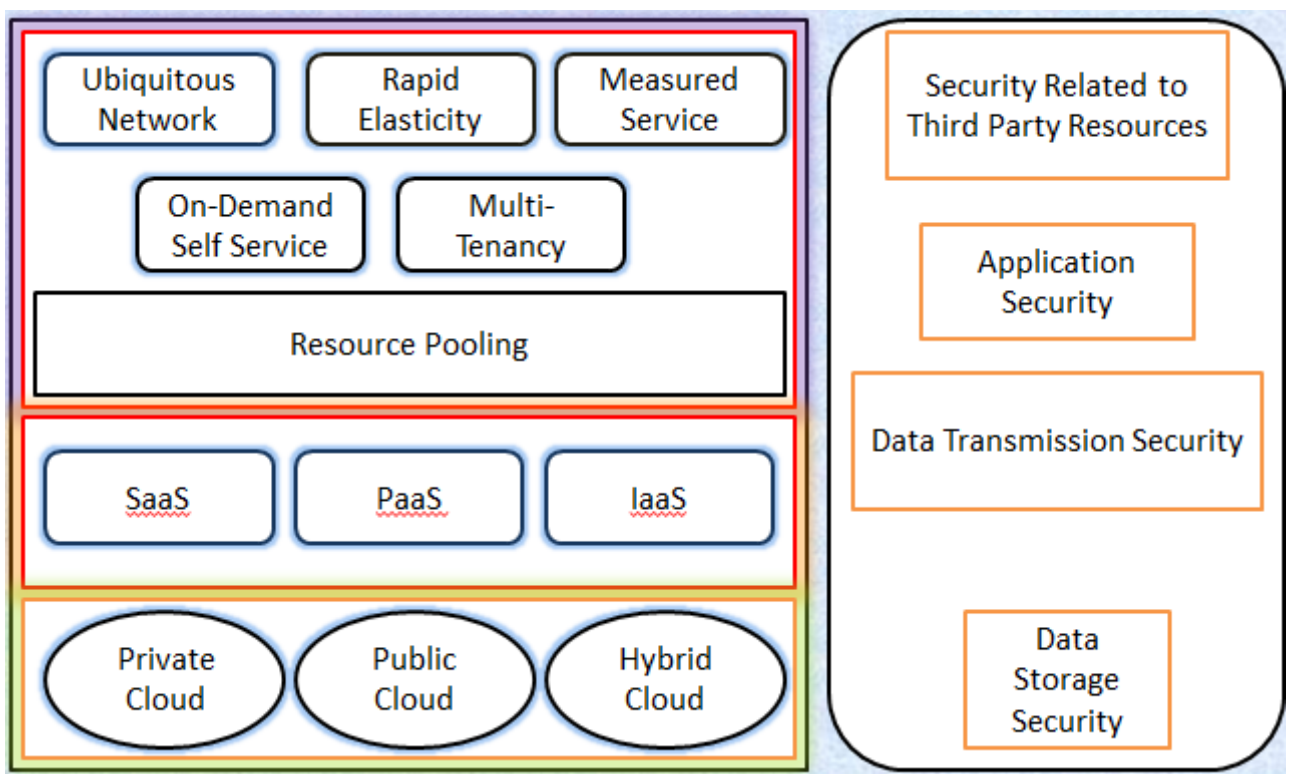

\section{A. Cloud Service Models}

The service models categorize vendors and the services provided according to Cloud Computing Architecture types. These cloud models form the core of the cloud and they exhibit characteristics depicted in the above layer. There are precisely three defined service models for the cloud platform.

- Cloud Software as a Service (SaaS): The capability provided to the consumer is to use the provider's application running on cloud infrastructure. The applications are accessible from various client devices through a thin client interface such as a web browser (e.g., web based email). The consumer does not manage or control the underlying cloud infrastructure including network, servers, operating systems, storage or even individual application capabilities, with the possible exception of limited user-specific application configuration settings.

- Cloud Platform as a Service (PaaS): The capability provided to the consumer is to deploy onto the cloud infrastructure consumer created or acquired applications created using programming languages and tools supported by the provider. The consumer does not manage or control the underlying cloud infrastructure including network, servers, operating systems, or storage, but has control over the deployed applications and possibly application hosting environment configurations.

- Cloud Infrastructure as a Service (IaaS): The capability provided to the consumer is to provision processing, storage, networks, and other fundamental computing resources where the consumer is able to deploy and run arbitrary software, which can include operating systems and applications. The consumer does not manage or control the underlying cloud infrastructure but has control over operating systems, storage, deployed applications and possibly limited control of select networking components (e.g., host firewalls).

\section{B. Cloud Deployment Models}

Four types of deployment models exist for a cloud computing platform. 


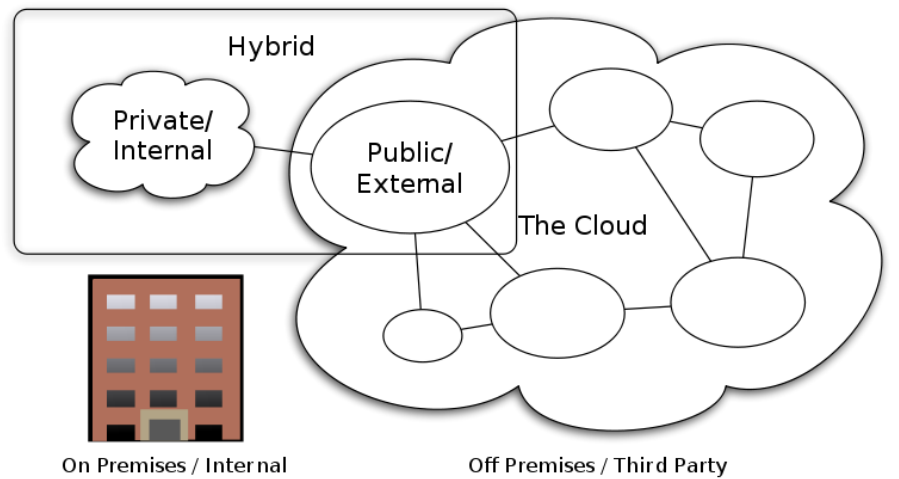

Cloud Computing Types

Fig 2. Deployment Models

- Private Cloud :The cloud infrastructure is operated solely for an organization. It may be managed by the organization or a third party and may exist on premise or off premise.

- Community Cloud: The cloud infrastructure is shared by several organizations and supports a specific community that has shared concerns (e.g., mission, security requirements, policy, and compliance considerations). It may be managed by the organizations or a third party and may exist on premise or off premise.

- Public Cloud: The cloud infrastructure is made available to the general public or a large industry group and is owned by an organization selling cloud services.

- Hybrid Cloud: The cloud infrastructure is a composition of two or more clouds (private, community, or public) that remain unique entities but are bound together by standardized or proprietary technology that enables data and applications portability (e.g, cloud bursting for load balancing between clouds).

\section{A Comparison Between Traditional In-House Computing, Grid and Cloud Computing}

In-House Computing: Traditional In-house computing is the concept of locally housing and maintaining the resources by the users themselves. Until the emergence and the recent popularity of cloud computing, in-house computing was the only means of utilizing resources. For example, a company who takes the in-house computing approach, may buy, install and maintain necessary hardware including the networking components such as servers. Also, they will install necessary system and application software in each computer. They will usually have dedicated administrators or IT staff for the maintenance of the whole computing environment

\section{Difference between In-House and Cloud Computing.}

Cloud computing has many advantages over in-house computing.

- Cloud computing is cheaper compared to in-house computing because there is minimum initial setup fees.

- Maintenance costs for in-house computing facilities can increase over its lifetime compared to the fixed costs of cloud computing services.

- Cloud computing facilities are highly scalable compared to in-house.

- It is very difficult and costly to maintain a supporting crew for the in-house computing facilities, cloud computing facilities always include the support of a set of systems, applications and database experts.

- It is easier to support a geographically dispersed and mobile workforce with clouds compared to in-house computing.

- Amidst all these advantages of using cloud computing, one reason of concern is its security. Cloud computing security is still an ongoing research area and cloud security and cloud access security have become highly active areas of discussion recently.

Grid Computing: Sharing of tasks over number of computers is known as Grid computing. The tasks can simply be data storage or it can be complex calculations. The distribution of tasks can be over large distances. The computers in a grid can act as a part of grid while they are not in use. In order to complete projects, the grid search for unutilized cycles on different computers to access them. One of the popular grid computing projects is SETI@home. There are many organizations that rely on different volunteers who offer their computers to be added on the grid. A virtual supercomputer is created after these computers are joined together. The principles of grid computing provide the way for modern 
supercomputers with many small computers attached to each other to form a supercomputer.

\section{Difference between Grid Computing and Cloud Computing}

- Cloud computing involves the use of services on the internet rather than local computers while grid computing involves sharing of tasks over multiple computers.

- Resources of multiple computers are shared in grid computing which greatly helps in improving the flexibility and power of the network whereas this not the case with cloud computing.

- Applications like spreadsheets, presentations, email and word processors are part of cloud computing whereas in grid computing, data storage or complex calculations are done.

\section{CONCLUSION}

Presently multi day, huge data is one of the greatest basic rising innovations. $4 \mathrm{v}$ 's of enormous records makes the data control and investigation requesting conditions for the customary measurements stockrooms. Various scientists verified that distributed computing is a top notch vehicle for net site facilitating enormous insights remaining burdens. In any case, running on gigantic information inside the cloud has its central goal of accommodating opposing format principles.

In this paper, we examined the benchmarks, conceivable outcomes, and horrendous circumstances of the cloud and huge actualities.

\section{REFERENCE}

[1] Jianfeng Yang and Zhibin Chen, " Cloud Computing Research and Security Issues”, IEEE 2010.

[2] [2]. S. Subashini and V. Kavitha, "A survey on security issues in service delivery models of cloud computing", Journal of Network and Computer Applications, vol. 34(2010) pages (1-11).

[3] [3]. Kassidy Clark, Martijn Warnier and Frances M.T. Brazier, "BOTCLOUDS - The Future of Cloud-based Botnets?"

[4] [4]. Peter Mell and Timothy Grace, " The NIST Definition of Cloud Computing (Draft)", Recommendations of the National Institute of Standards and Technology, 7 pages (January.2011).

[5] [5]. M.Tariq Banday, Jameel A. Qadri and Nisar A. Shah, "Study of Botnets and Their Threats to Internet Security", Sprouts - Working Papers on Information Systems.
[6] [6]. Moheeb Abu Rajab, Jay Zarfoss, Fabian Monrose and Andreas Terzis, “ A Multifaceted Approach to Understanding the Botnet Phenomenon".

[7] http://en.wikipedia.org/wiki/Cloud_computing

[8] Degado R., https://datafloq.com/read/opportunitieschallenges-cloudcomputing-big-data/4456

[9] Robinson S., (2012, 2015), - The Storage and Transfer Challenges of Big Data.Accessed\|, http://sloanreview.mit.edu/article/the-storage-andtransferchallenges-of-big-data/.

[10] Chen J., Chen Y., Du X., Li C., Lu J., Zhao S., and Zhou X.., (2013), - Big Data Challenge: A Data Management Perspective.|l Frontiers of Computer Science 7 (2) (Pg157-164).

[11] Chen M., Mao S., Zhang Y., and Leung V. C.. (2014), -Chapter 1, Big Data: Related Technologies, Challenges, and Future Prospects\|, Heidelberg: Springer 